\title{
Near-Earth asteroids among the lota Aquariids meteoroid stream
}

\author{
1 Institute of Astrophysics, Dushanbe 734042, Tajikistan \\ e-mail: P.B.Babadzhanov@mail.ru \\ 2 Queen Mary University of London, E1 4NS, UK \\ e-mail: I.P.Williams@qmul.ac.uk
}

P. B. Babadzhanov ${ }^{1}$, I. P. Williams ${ }^{2}$, and G. I. Kokhirova ${ }^{1}$

Received 20 July 2009 / Accepted 10 September 2009

\begin{abstract}
It is shown that the near-Earth asteroids 2002JS2, 2002PD11, and 2003MT9 have very similar orbits to each other and probably a common origin. We investigate the orbital evolution of these asteroids under the perturbing action of the planets and calculated the theoretical shower parameters for any meteor shower that may be associated with these asteroids. Finally we searched the existing catalogues of meteor showers and fireballs, and found that activity has been observed corresponding to each of the theoretically predicted shower. We conclude that this asteroid-meteoroid complex is the result of a cometary break-up.
\end{abstract}

Key words. meteors, meteoroids - minor planets, asteroids

\section{Introduction}

In recent years there has been a significant amount of popular interest in near-Earth objects (NEOs) because of the potential threat that they represent to the Earth through collisions. By definition, an NEO has an orbit with a perihelion distance that is short enough to allow possible close approaches to the Earth. Lists of known NEOs, together with their orbital elements, can be found on web sites, and a review of their properties is given by Binzel \& Lupishko (2006). Since the dynamical lifetimes of objects on such orbits are less than 10 Myr (Morbidelli \& Gladman 1998), they must have originated in populations of bodies currently found in the Solar System.

The two most obvious populations are the main-belt asteroids and short-period comets. The difference in composition between these two populations suggests that mitigation strategy that might be employed is rather different, depending on which of the two possible origins the particular threatening object had. The first is primarily composed of stone and metal while the second is primarily ice. Their internal strength and vaporization temperature are different, implying a rather different reaction to the various proposed mitigation strategies.

For many objects, distinguishing between comets and asteroids is easy, a comet becomes active when close to the Sun, forming a visible tail while an asteroid shows no signs of such an activity. However, a dormant comet nucleus may be covered by a thick dust mantle, thus making its appearance through telescopes very similar to that of an asteroid. The dust mantle may also prevent sublimation so that activity ceases. The problem of distinguishing between a dormant comet and an asteroid is not confined to the NEO population, as discussed for example in the outer asteroid belt by Fitzsimmons et al. (1994), Williams (1997). Near the end of their lives, a comet nucleus may also fragment, leaving several small objects on very similar orbits to each other (Jenniskens \& Vaubaillon 2008), none showing any signs of cometary activity and all classed as asteroids.
There have been numerous attempts to determine the fraction of extinct comets among the NEO population, for example by considering properties such as density and shape. In this paper we take a different approach. Whipple (1951) first showed that sublimation of cometary ices would cause an outflow of gasses that could carry small grains out with it through drag forces. Such grains would have a speed relative to the comet nucleus that is much less than the orbital speed and so would move on similar orbits to the parent, thus forming a meteoroid stream. Such a meteoroid stream would be observed on Earth as a meteor shower provided one of the nodal distances of the stream was close to $1 \mathrm{AU}$, since the Earth could then pass through stream. This has been regarded as the main mechanism of stream formation since that date (see e.g. Williams 2001). However, Jenniskens (2004) suggested that the fragmentation of a comet nucleus could also lead to the formation of a stream, thus reviving earlier theories based on the observations of comet Biela in 1846 (for a discussion of early progress, see Williams 2004). The relationship between 3D/Biela and the Andromedids is discussed in Jenniskens \& Vaubaillon (2007). It is also possible that a meteoroid stream can be formed through mutual collisions between asteroids. This subject was reviewed by Obrubov (1999). Williams (1993) claimed that streams formed in this way would contain far less mass and be far more diffuse than those from cometary origin. There are a number of NEOs that have similar orbits to meteoroid streams. The similarity between the orbits of the Taurid meteoroid complex and a number of asteroids was first discussed by Steel et al. (1991). Porubcan et al. (2004) reviewed the whole subject of asteroids moving on similar orbits to meteoroid streams, though at that time, with the exception of the Taurid complex, they found no more than one asteroid associated with any given stream. The Taurid complex has also been revised by Porubcan et al. (2006), Babadzhanov et al. (2008a). Since the review of Porubcan et al. (2004), there have been a number of advances, the most notable perhaps being NEO 2003EH1 and its association 
with the Quadrantid shower (Jenniskens 2004; Williams et al. 2004), while Babadzhanov \& Williams (2007) found meteor showers associated with 2000PG3 and Babadzhanov et al. (2008b) found that 4 NEOs were related to the Piscids meteoroid stream. Jenniskens \& Vaubaillon (2008) showed that the Kappa Cygnids had a similar origin. An overview of the topic is given in Jenniskens (2008). We contend that the vast majority of NEOs found on orbits similar to those of meteoroid streams formed through the fragmentation of a cometary nucleus as discussed by Jenniskens \& Vaubillion (2008) and that only a tiny minority arises through inter asteroid collisions.

We thus assume that any NEO moving on an orbit similar to those of comets and have a meteoroid stream associated with it is likely to be of cometary origin and that this can be a meaningful test of the probable cometary nature of an NEO. Where there are multiple asteroids moving on a similar orbit to a meteoroid stream, the above conclusion would appear to be even stronger. Hence, in this paper we search through the list of known NEOs for sets of objects moving on similar orbits. Having located such a set, we investigate whether or not there are meteor showers that might be associated with this set.

\section{A search for NEOs moving on similar heliocentric orbits to each other}

The orbital elements of 3143 NEOs known on 2005 January 1 can be found at http://newton.dm.unipi.it/neodys/ neodys. cat, and this data set forms the basis of our search for groups of NEOs with orbits that are similar to each other. An important tool for quantifying the differences between two orbits was developed by Southworth \& Hawkins (1963) and is based on the square of the differences between the orbital elements in phase-space. The expression they found, $D_{\mathrm{S}-\mathrm{H}}$, is given by

$$
\begin{aligned}
D_{\mathrm{S}-\mathrm{H}}^{2}= & \left(e_{2}-e_{1}\right)^{2}+\left(q_{2}-q_{1}\right)^{2}+\left(2 \sin \frac{i_{2}-i_{1}}{2}\right)^{2} \\
& +\sin i_{1} \sin i_{2}\left(2 \sin \frac{\Omega_{2}-\Omega_{1}}{2}\right)^{2} \\
& +\left[\left(\frac{e_{1}+e_{2}}{2}\right) 2 \sin \frac{\left(\Omega_{2}+\omega_{2}\right)-\left(\Omega_{1}+\omega_{1}\right)}{2}\right]^{2},
\end{aligned}
$$

where subscripts 1 and 2 relate to these two orbits that are being compared, $e$ is the eccentricity, $q=a(1-e)$ is the perihelion distance, $a$ is the semi-major axis, $i$ is the inclination, $\Omega$ is the longitude of ascending node, and $\omega$ is the argument of perihelion. A value of $D_{\mathrm{S}-\mathrm{H}} \leq 0.20$ is generally taken to indicate similarity between the two orbits.

The longitude of the ascending node $\Omega$ and the argument of perihelion $\omega$ change periodically over a reasonable time scales so that $D_{\mathrm{S}-\mathrm{H}}$ can become large simply by these changes. This makes the above criterion unsuitable if orbits are to be compared over a long time interval. Asher et al. (1993) proposed a simplified $D$ criterion that avoided this, namely

$D^{2}=\left(\frac{a_{1}-a_{2}}{3}\right)^{2}+\left(e_{1}-e_{2}\right)^{2}+\left\{2 \sin \left[\left(i_{1}-i_{2}\right) / 2\right]\right\}^{2}$,

which is more acceptable for orbital similarity of two orbits. The procedure generally used to find meteor streams in a data set is based upon an iterative procedure used by Sekanina (1970). Full details can be found also in Arter \& Williams (1997). However as the numbers of NEOs with a similar orbit is likely to be small a simplified version can be used. By looking at Eq. (2), we see
Table 1. The values of the simplified $D$ criterion.

\begin{tabular}{lccc}
\hline \hline Asteroid & 2002JS2 & 2002PD11 & 2003MT9 \\
\hline 2002JS2 & 0.00 & 0.04 & 0.16 \\
2002PD11 & 0.04 & 0.00 & 0.11 \\
2003MT9 & 0.16 & 0.11 & 0.00 \\
\hline
\end{tabular}

that a necessary condition for $D<0.2$ is that the difference in eccentricity is less than 0.2. Similarly the difference in semimajor axis has to be less than 0.6. With such filters it is relatively straight forward to identify sets of NEOs satisfying the criterion that $D$ as given by Eq. (2) is less than 0.2. We found that the 3 asteroids, 2002JS2, 2002PD11, and 2003MT9 have differences in orbital elements that were less than the minimum separation laid down (i.e. $D<0.2$ ) and so may be assumed to be associated with each other.

The calculated values of the $D$ criterion for the relative pairings of these objects are given in Table 1. It can be seen that the value of the $D$ for any pair of these asteroids range from 0.04 to 0.16 indicating strongly that all are on very similar orbits.

The orbital elements of these three asteroids are given in Table 2 (equinox 2000.0). We also give other useful information about these asteroids in the same table. $R_{\mathrm{a}}$ and $R_{\mathrm{d}}$ are the nodal distances of the ascending and descending nodes at the present time. From these values we see that if a meteoroid stream had these current values, it would not produce a meter shower as the Earth does not intersect the current orbit.

An other useful parameter is $T_{j}$ is the Tisserand invariant and is given relative to Jupiter by

$T_{j}=\frac{a_{j}}{a}+2\left[\frac{a}{a_{j}}\left(1-e^{2}\right)\right]^{0.5} \cos i$,

where $a_{j}=5.2 \mathrm{AU}$ is the semi-major axis of the Jupiters orbit. Kresak (1954) originally suggested that for comets $2.08<T_{j} \leq$ 3.12 , and for asteroids $T_{j}>3.12$. These limits have been slightly modified, but a value of $T_{j}$ at around 3 is still considered to be the boundary between asteroidal and cometary orbits. The values of $T_{j}$ are also given in Table 2. As can be seen, the last two asteroids are moving on comet like orbits.

The final two parameters given in Table 2 are the absolute magnitude $H$ and the diameter $d$ of each asteroid. The diameter was calculated using the expression (Bowell \& Lumme 1982)

$\log d=10^{3.12-0.2 H} / \sqrt{p}$,

where $p$ is the albedo. Asteroids of cometary origin are very dark and have very low values of their albedo, usually in the range from 0.02 to 0.12 (Jewitt 1992). In Table 2, the values of $d$ were obtained assuming an albedo of 0.07 .

\section{The orbital evolution of the NEOs}

As mentioned already, the orbits of NEOs evolve on a rapid timescale because of their location in the Solar system. In most cases this evolution thus can be represented as a periodic change with minor oscillations about a sinusoidal curve. This oscillation was called "nutation cycle" in Jenniskens (2006). Examples of such evolution can be found in Wu \& Williams (1992), Williams \& Wu (1993), Babadzhanov et al. (2008a,b).

As meteoroids are ejected from their parent body with a range of different velocities (all, as mentioned earlier, much less than the orbital velocities) they will initially move on orbits that are similar, but not identical to that of the parent. Any difference 
Table 2. Orbital elements of the three near-Earth asteroids.

\begin{tabular}{lcccccccccccc}
\hline \hline Asteroid & $\begin{array}{c}a \\
\mathrm{AU}\end{array}$ & $e$ & $q$ & $i^{\circ}$ & $\Omega^{\circ}$ & $\omega^{\circ}$ & $\pi^{\circ}$ & $H$ & $\begin{array}{c}d \\
\mathrm{~km}\end{array}$ & $\begin{array}{c}R_{\mathrm{a}} \\
\mathrm{AU}\end{array}$ & $\begin{array}{c}R_{\mathrm{d}} \\
\mathrm{AU}\end{array}$ & $T_{j}$ \\
& & $\mathrm{AU}$ & & & & & & & \\
\hline 2002JS2 & 2.131 & 0.825 & 0.379 & 11.5 & 124.0 & 320.1 & 84.1 & 17.57 & 1.53 & 0.42 & 1.86 & 3.14 \\
2002PD11 & 2.226 & 0.863 & 0.304 & 11.6 & 298.5 & 149.0 & 87.5 & 19.97 & 0.51 & 2.18 & 0.32 & 2.98 \\
2003MT9 & 2.520 & 0.920 & 0.202 & 6.8 & 233.2 & 200.7 & 73.9 & 18.53 & 0.98 & 2.78 & 0.21 & 2.62 \\
\hline
\end{tabular}

$(0,0)$

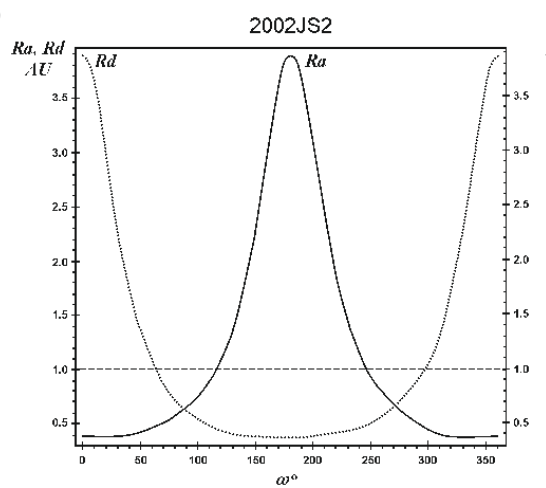

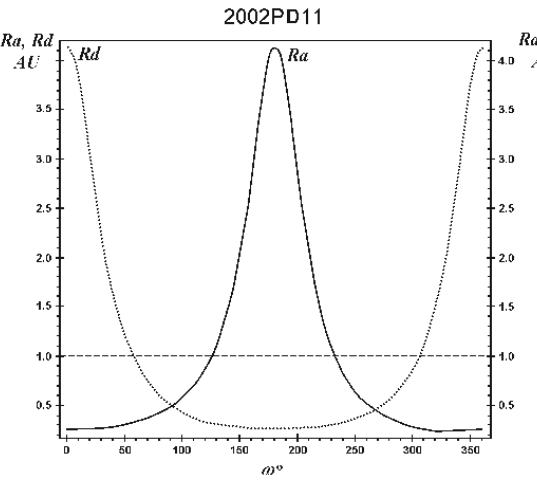

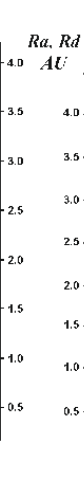

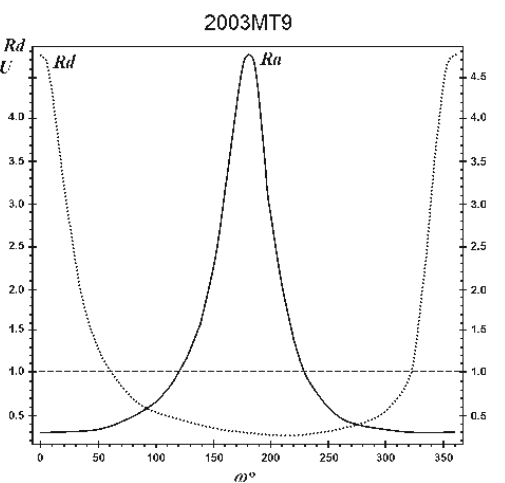

Fig. 1. The heliocentric distance of the ascending node $R_{\mathrm{a}}$ and descending node $R_{\mathrm{d}}(--)$ plotted against the argument of perihelion $\omega$ for each of the NEAs 2002JS2, 2002PD11, and 2003MT9.

in the semi-major axes is reflect in a corresponding change in the orbital periods between the meteoroids and their parent body so that some meteoroids lag behind the parent body more and more, while others, overtaking it, spread along the entire orbit and form a complete loop in a comparatively short time (Williams 1995). Because the meteoroids occupy various positions relatively of the parent body, particularly in true anomaly, in any given time, they experience different planetary perturbations and so have differing rates of orbital evolution. This means in particular that the rates and cycles of variations in the angular orbital elements (the argument of perihelia $\omega$, the longitude of the ascending node $\Omega$, and the inclination to the ecliptic $i$ ) will be different for different meteoroids. As a result, within one such cycle, the stream meteoroids will be found on orbits similar to all evolutionary tracks of their parent body.

In order to produce a meteor shower, the Earth must intersect the meteoroid stream. The Earths orbit can only be intersected by those stream meteoroids which have a nodal distance of about $1 \mathrm{AU}$, i.e. it should be fulfilled the following condition:

$\frac{q(1+e)}{1-e \cos \omega} \approx 1, \quad$ or $\quad \frac{q(1+e)}{1+e \cos \omega} \approx 1$.

In general there are 4 possible values of $\omega$ that are solutions of one or other of the above equations for any given pair of values of $q$ and $e$. As a result, the meteoroid stream might produce a night-time meteor shower with northern and southern branches at the pre-perihelion intersection with the Earth, and a day-time twin meteor shower with northern and southern branches at the post-perihelion intersection with the Earth or of course the reverse as was first shown by Babadzhanov \& Obrubov (1992). They also showed that if the evolution in $a$ and $e$ was significant 8 intersections could exist. In the past, for the majority of such cases, the branches have been regarded as independent showers produced by their own meteoroid stream rather than being a product of one perturbed stream. The reason for this is obvious, meteor showers have been observed long before its nature was understood and meteoroid streams recognized. A good example of the above is the day-time meteor showers $\zeta$-Perseids and $\beta$-Taurids that are in reality the northern and southern branches of the day-time shower produced by the Taurid meteoroid stream.

In order to determine the locations of these intersections with the Earth's orbit, it is necessary to know the values of $q$ and $e$ at all times during one cycle of variation of the argument of perihelion. To obtain these values, it is necessary to integrate the orbit of the parent body back in time. Any of the numerical methods currently in use to follow the formation and evolution of a meteoroid stream (e.g. Wu \& Williams 1993; Vaubaillon et al. 2005) are suitable, but the computational effort involved and the resulting level of detail produced varies from method to method. Hence the most efficient method to use depends on the results required and the type of orbit. For the two NEOs NEAs 2002JS2 and 2002PD11, we have used the Halphen-Goryachev integration method (Goryachev 1937) to calculate the secular variations of the orbital elements as this was shown by Babadzhanov (2001) to be quite efficient for integrating this type of orbits. NEA 2003MT9 moves on an orbit that is in 3:1 resonance with Jupiter. This potentially makes secular variational methods unsuitable for integration since the method depends on averaging the perturbations over one orbit. When resonance is present certain configurations can occur more frequently than others, making averaging over an orbit difficult. Hence, the computationally more expensive method of Everhart (1974) was used, with gravitational perturbations from the eight planets and Pluto being included, though it turns out that the evolution is very similar to that of the other two NEA's. The nutation cycle equal to one cycle of variation of the argument of perihelion for NEAs 2002JS2, 2002PD11, and 2003MT9 is 9625, 7350, and probably more than 12000 years respectively.

In Fig. 1 we show the dependences of the heliocentric distances of the ascending node $R_{\mathrm{a}}$ and descending node $R_{\mathrm{d}}$ against the argument of perihelion over one cycle of the variation in the argument of perihelion for each of the 3 asteroids. From these figures we see that each of these asteroids intersects the Earths orbit four times during one such cycle. It is also interesting to note that for each of the asteroids the relevant values of $\omega$ are very similar. In fact all the crossings take place at locations where the value of $\omega$ lies within the following 
Table 3. Meteor showers associated with the NEA 2002JS2.

\begin{tabular}{|c|c|c|c|c|c|c|c|c|c|c|c|c|c|c|}
\hline $\begin{array}{l}\text { Meteor } \\
\text { showers }\end{array}$ & $\begin{array}{c}q \\
\mathrm{AU}\end{array}$ & $e$ & $i^{\circ}$ & $\Omega^{\circ}$ & $\overline{\overline{\omega^{\circ}}}$ & $\overline{\pi^{\circ}}$ & $\overline{L_{\odot}^{\circ}}$ & Date & $\alpha^{\circ}$ & $\delta^{\circ}$ & $\begin{array}{c}V_{\mathrm{g}} \\
\mathrm{km} \mathrm{s}^{-1}\end{array}$ & $\overline{D_{\mathrm{S}-\mathrm{H}}}$ & $\begin{array}{l}\text { Ty } \\
\text { pe }\end{array}$ & $\begin{array}{l}\text { Cata- } \\
\text { logue }\end{array}$ \\
\hline 2002JS2(A) & .365 & .829 & 8.4 & 150.3 & 293.8 & 84.1 & 150.3 & Aug. 23 & 340.8 & -0.4 & 28.1 & & $\mathbf{N}$ & \\
\hline 33 NIA & .277 & .836 & 1.4 & 136.7 & 307.5 & 84.2 & 136.7 & Aug. 09 & 336.2 & -8.8 & 28.8 & .15 & $\mathrm{~N}$ & S2 \\
\hline 33 NIA & .249 & .762 & 4.4 & 138.0 & 319.1 & 97.1 & 147.7 & Aug. 20 & 343.1 & -3.4 & 24.5 & .24 & $\mathrm{~N}$ & S3 \\
\hline 33 NIA & .256 & .929 & 5.8 & 130.0 & 306.6 & 76.6 & 130.0 & Aug. 03 & 327.5 & -9.3 & 34.4 & .19 & $\mathrm{~N}$ & G \\
\hline 33 NIA & .358 & .852 & 7.4 & 145.1 & 297.4 & 82.5 & 147.7 & Aug. 20 & 328.0 & -4.7 & 27.6 & .04 & $\mathrm{~N}$ & DMS \\
\hline 33 NIA & .238 & .934 & 5.7 & 129.5 & 306.7 & 96.4 & 147.7 & Aug. 20 & 326.9 & -9.3 & 34.4 & .22 & $\mathrm{~N}$ & PG \\
\hline 124 meteors & .322 & .793 & 8.9 & 145.3 & 304.1 & 89.4 & 145.3 & Aug. 18 & 341.0 & -0.4 & 28.8 & .10 & $\mathrm{~N}$ & MODC \\
\hline & \pm .010 & \pm .006 & \pm .6 & \pm .6 & \pm 1.4 & \pm 1.1 & & & \pm .5 & \pm .5 & \pm .3 & & & \\
\hline 2002JS2(B) & .336 & .842 & 6.7 & 327.0 & 117.1 & 84.1 & 147.0 & Aug. 20 & 343.7 & -12.8 & 28.9 & & $\mathbf{N}$ & \\
\hline 3 SIA & .208 & .912 & 6.9 & 311.7 & 131.8 & 83.5 & 131.7 & Aug. 04 & 334.0 & -14.4 & 33.8 & .15 & $\mathrm{~N}$ & $\mathrm{C}$ \\
\hline 3 SIA & .254 & .880 & 8.0 & 307.7 & 126.0 & 73.7 & 127.7 & July 31 & 328.7 & -17.8 & 31.0 & .19 & $\mathrm{~N}$ & $\mathrm{~K}$ \\
\hline 3 SIA & 190 & .929 & 8.6 & 306.9 & 137.5 & 84.4 & 131.7 & Aug. 04 & 339.0 & -15.6 & 34.8 & .18 & $\mathrm{~N}$ & DMS \\
\hline 95 meteors & .327 & .805 & 7.7 & 321.2 & 122.3 & 83.5 & 141.2 & Aug. 14 & 340.1 & -14.7 & 29.3 & .05 & $\mathrm{~N}$ & MODC \\
\hline & \pm .011 & \pm .007 & \pm .6 & \pm .5 & \pm 1.5 & \pm 1.1 & & & \pm .8 & \pm .4 & \pm .3 & & & \\
\hline 2002JS2(C) & .332 & .844 & 6.6 & 21.2 & 62.9 & 84.1 & 21.6 & Apr. 11 & 5.2 & 8.0 & 29.0 & & $\overline{\mathbf{D}}$ & \\
\hline 144 APS & .224 & .830 & .5 & 30.3 & 45.4 & 75.7 & 30.3 & Apr. 21 & 7.6 & 3.3 & 28.9 & .20 & D & K \\
\hline 144 APS & .341 & .780 & 6.0 & 30.7 & 59.0 & 89.7 & 30.7 & Apr. 21 & 12.6 & 10.3 & 26.8 & .10 & D & $\mathrm{K}$ \\
\hline 2002JS2(D) & .363 & 830 & 8.1 & 197.7 & 246.4 & 84.1 & 17.7 & Apr. 07 & 8.1 & -4.1 & 28.1 & & $\mathbf{D}$ & \\
\hline 419 DAC & .274 & .830 & 11.0 & 209.7 & 233.0 & 82.7 & 29.7 & Apr. 20 & 13.6 & -2.7 & 28.9 & .11 & $\mathrm{D}$ & K \\
\hline
\end{tabular}

$\mathrm{D}$ and $\mathrm{N}$ denote day- and night-time activity respectively.

Table 4. Meteor showers associated with the NEA 2002PD11.

\begin{tabular}{|c|c|c|c|c|c|c|c|c|c|c|c|c|c|c|}
\hline $\begin{array}{l}\text { Meteor } \\
\text { showers }\end{array}$ & $\begin{array}{c}q \\
\mathrm{AU}\end{array}$ & $e$ & $i^{\circ}$ & $\Omega^{\circ}$ & $\omega^{\circ}$ & $\pi^{\circ}$ & $L_{\odot}^{\circ}$ & Date & $\alpha^{\circ}$ & $\delta^{\circ}$ & $\begin{array}{c}V_{\mathrm{g}} \\
\mathrm{km} \mathrm{s}^{-1}\end{array}$ & $D_{\mathrm{S}-\mathrm{H}}$ & $\begin{array}{l}\text { Ty } \\
\text { pe }\end{array}$ & $\begin{array}{l}\text { Cata- } \\
\text { logue }\end{array}$ \\
\hline 2002PD11(A) & .325 & .854 & 7.2 & 150.0 & 297.4 & 87.4 & 150.0 & Aug. 23 & 342.4 & -1.5 & 29.5 & & $\mathbf{N}$ & \\
\hline 33 NIA & .277 & .836 & 1.4 & 136.7 & 307.5 & 84.2 & 136.7 & Aug. 09 & 336.2 & -8.8 & 28.8 & .12 & $\mathrm{~N}$ & S2 \\
\hline 33 NIA & .249 & .762 & 4.4 & 138.0 & 319.1 & 97.1 & 147.7 & Aug. 20 & 343.1 & -3.4 & 24.5 & .19 & $\mathrm{~N}$ & S3 \\
\hline 33 NIA & .256 & .929 & 5.8 & 130.0 & 306.6 & 76.6 & 130.0 & Aug. 03 & 327.5 & -9.3 & 34.4 & .19 & $\mathrm{~N}$ & G \\
\hline 33 NIA & .358 & .852 & 7.4 & 145.1 & 297.4 & 82.5 & 147.7 & Aug. 20 & 328.0 & -4.7 & 27.6 & .08 & $\mathrm{~N}$ & DMS \\
\hline 33 NIA & .238 & .934 & 5.7 & 129.5 & 306.7 & 96.4 & 147.7 & Aug. 20 & 326.9 & -9.3 & 34.4 & .22 & $\mathrm{~N}$ & \\
\hline 128 meteors & .294 & .813 & 9.0 & 145.0 & 306.9 & 61.1 & 145.0 & Aug. 18 & 342.1 & -0.6 & 29.7 & .09 & $\mathrm{~N}$ & MODC \\
\hline & \pm .010 & \pm .006 & \pm .6 & \pm .6 & \pm 1.4 & \pm 1.1 & & & \pm .5 & \pm .4 & \pm .3 & & & \\
\hline 2002PD11(B) & .292 & .869 & 7.3 & 325.9 & 121.5 & 87.4 & 145.9 & Aug. 19 & 344.5 & -12.2 & 30.6 & & $\mathbf{N}$ & \\
\hline 3 SIA & .208 & .912 & 6.9 & 311.7 & 131.8 & 83.5 & 131.7 & Aug. 04 & 334.0 & -14.4 & 33.8 & .12 & $\mathrm{~N}$ & $\mathrm{C}$ \\
\hline SIA & .190 & .929 & 8.6 & 306.9 & 137.5 & 84.4 & 131.7 & Aug. 04 & 339.0 & -15.6 & 34.8 & .14 & $\mathrm{~N}$ & DMS \\
\hline 75 meteors & .272 & .824 & 9.8 & 322.1 & 128.1 & 90.2 & 142.1 & Aug. 15 & 344.4 & -13.6 & 31.3 & .08 & $\mathrm{~N}$ & MODC \\
\hline & \pm .010 & \pm .013 & \pm .8 & \pm .6 & \pm 1.4 & \pm 1.0 & & & \pm .7 & \pm .5 & \pm .4 & & & \\
\hline 2002PD11(C) & .287 & .871 & 7.1 & 29.4 & $\mathbf{5 8 . 0}$ & 87.4 & 29.4 & Apr. 20 & 10.9 & 10.1 & 30.7 & & D & \\
\hline 144 APS & .224 & .830 & .5 & 30.3 & 45.4 & 75.7 & 30.3 & Apr. 21 & 7.6 & 3.3 & 28.9 & .22 & D & K \\
\hline 144 APS & .341 & .780 & 6.0 & 30.7 & 59.0 & 89.7 & 30.7 & Apr. 21 & 12.6 & 10.3 & 26.8 & .11 & D & \\
\hline 38 meteors & .369 & .791 & 6.8 & 27.6 & 63.3 & 90.9 & 27.6 & Apr. 18 & 10.5 & 11.3 & 28.2 & .12 & D & MODC \\
\hline & \pm .016 & \pm .009 & \pm .7 & \pm 1.1 & \pm 2.3 & \pm 1.5 & & & \pm .8 & \pm .7 & \pm .3 & & & \\
\hline 2002PD11(D) & .323 & .855 & 7.4 & 205.5 & 241.9 & 87.4 & 25.5 & Apr. 16 & 13.2 & -0.5 & 29.5 & & $\mathbf{D}$ & \\
\hline 419 DAC & .274 & .830 & 11.0 & 209.7 & 233.0 & 82.7 & 29.7 & Apr. 20 & 13.6 & -2.7 & 28.9 & .11 & $\mathrm{D}$ & \\
\hline meteors & .295 & .826 & 7.4 & 208.6 & 234.7 & 83.3 & 28.6 & Apr. 19 & 12.1 & -0.5 & 30.1 & .07 & $\mathrm{~N}$ & MODC \\
\hline & \pm .015 & \pm .009 & \pm .9 & \pm 1.1 & \pm 2.2 & \pm 1.4 & & & \pm .9 & \pm .7 & \pm .5 & & & \\
\hline
\end{tabular}

$\mathrm{D}$ and $\mathrm{N}$ denote day- and night-time activity respectively.

ranges: $58^{\circ}-63^{\circ}, 117^{\circ}-122^{\circ}, 222^{\circ}-246^{\circ}$, and $294^{\circ}-318^{\circ}$. Because all three NEOs produce four locations where crossing of their orbit with the Earths orbit occurs, it is theoretically possible that a meteoroid stream associated with any of these asteroids can produce two day-time and two night-time meteor showers. The orbital elements, the position of the radiant (right ascention $\alpha$ and declination $\delta$ ), the geocentric velocity $V_{\mathrm{g}}\left(\mathrm{km} \mathrm{s}^{-1}\right)$, the solar longitude $L_{\odot}$, and the corresponding dates of maximum activity, of the possible meteor showers associated with each of the three asteroids can be calculated from the data above. The results of these calculations are given in
Tables 3-5, where all values of the theoretical showers are in bold fonts (equinox 2000.0). The table also contains the corresponding data for possible observational counterpart, the derivation of which we describe in the next section.

\section{Search for meteor showers associated with NEOs 2002JS2, 2002PD11, and 2003 MT9}

The orbital elements of theoretical meteoroid streams associated with the three NEAs 2002JS2, 2002PD11, and 2003MT9 
Table 5. Meteor showers associated with the NEA 2003MT9.

\begin{tabular}{|c|c|c|c|c|c|c|c|c|c|c|c|c|c|c|}
\hline $\begin{array}{l}\text { Meteor } \\
\text { showers }\end{array}$ & $\begin{array}{c}q \\
\mathrm{AU}\end{array}$ & $e$ & $i^{\circ}$ & $\Omega^{\circ}$ & $\omega^{\circ}$ & $\pi^{\circ}$ & $L_{\odot}^{\circ}$ & Date & $\alpha^{\circ}$ & $\delta^{\circ}$ & $\begin{array}{c}V_{\mathrm{g}} \\
\mathrm{km} \mathrm{s}^{-1}\end{array}$ & $D_{\mathrm{S}-\mathrm{H}}$ & $\begin{array}{l}\text { Ty } \\
\text { pe }\end{array}$ & $\begin{array}{l}\text { Cata- } \\
\text { logue }\end{array}$ \\
\hline 2003МТ9(А) & .155 & .939 & 4.3 & 115.7 & 318.2 & 73.9 & 115.7 & July 18 & 319.2 & -13.8 & 36.3 & & $\mathbf{N}$ & \\
\hline 33 NIA & 211 & .890 & 12.0 & 120.7 & 313.0 & 73.7 & 120.7 & July 23 & 321.7 & -7.8 & 33.2 & .15 & $\mathrm{~N}$ & $\mathrm{~K}$ \\
\hline 33 NIA & .256 & .929 & 5.8 & 130.0 & 306.6 & 76.6 & 130.0 & Aug. 03 & 327.5 & -9.3 & 34.4 & .12 & $\mathrm{~N}$ & G \\
\hline 33 NIA & .238 & .934 & 5.7 & 129.5 & 306.7 & 96.4 & 147.7 & Aug. 20 & 326.9 & -9.3 & 34.4 & .10 & $\mathrm{~N}$ & \\
\hline 81 meteors & $\begin{array}{c}.189 \\
\pm .011\end{array}$ & $\begin{array}{c}.888 \\
\pm .016\end{array}$ & $\begin{array}{r}12.4 \\
\pm 1.1\end{array}$ & $\begin{array}{c}115.6 \\
\pm .8\end{array}$ & $\begin{array}{c}316.9 \\
\pm 1.7\end{array}$ & $\begin{array}{r}72.5 \\
\pm 1.1\end{array}$ & 115.6 & July 18 & $\begin{array}{c}318.4 \\
\pm .6\end{array}$ & $\begin{array}{c}-10.8 \\
\pm .4\end{array}$ & $\begin{array}{c}35.6 \\
\pm .4\end{array}$ & .16 & $\mathrm{~N}$ & MODC \\
\hline 2003МT9(B) & .291 & .883 & 1.7 & 313.3 & 120.6 & 73.9 & 133.3 & Aug. 06 & 330.3 & -13.4 & 31.1 & & $\overline{\mathbf{N}}$ & \\
\hline 3 SIA & .254 & .880 & 8.0 & 307.7 & 126.0 & 73.7 & 127.7 & July 31 & 328.7 & -17.8 & 31.0 & .12 & $\mathrm{~N}$ & K \\
\hline 3 SIA & .212 & 917 & 7.2 & 312.7 & 130.7 & 83.4 & 132.7 & Aug. 05 & 334.7 & -14.7 & 34.0 & .20 & $\mathrm{~N}$ & $\mathrm{C} 1$ \\
\hline 3 SIA & 190 & .929 & 8.6 & 306.9 & 137.5 & 84.4 & 131.7 & Aug. 04 & 339.0 & -15.6 & 34.8 & .23 & $\mathrm{~N}$ & DMS \\
\hline 97 meteors & $\begin{array}{c}.274 \\
\pm .011\end{array}$ & $\begin{array}{c}.834 \\
\pm .010\end{array}$ & $\begin{array}{l}6.9 \\
\pm .6\end{array}$ & $\begin{array}{c}310.0 \\
\pm .8\end{array}$ & $\begin{array}{l}127.8 \\
\pm 1.5\end{array}$ & $\begin{array}{l}77.8 \\
\pm 1.1\end{array}$ & 130.0 & Aug. 02 & $\begin{array}{c}331.6 \\
\pm .6\end{array}$ & $\begin{array}{c}-16.4 \\
\pm .3\end{array}$ & $\begin{array}{c}31.2 \\
\pm .3\end{array}$ & .12 & $\mathrm{~N}$ & MODC \\
\hline 2003МТ9(С) & .304 & .878 & 1.8 & 13.1 & 60.8 & 73.9 & 13.1 & Apr. 03 & 358.9 & .9 & 30.6 & & $\overline{\mathbf{D}}$ & \\
\hline 144 APS & .224 & .830 & .5 & 30.3 & 45.4 & 75.7 & 30.3 & Apr. 21 & 7.6 & -2.7 & 28.9 & .10 & D & K \\
\hline 2003MT9(D) & .155 & .938 & 4.4 & 212.3 & $\begin{array}{l}221.7 \\
\text { Meteo }\end{array}$ & $\begin{array}{c}74.0 \\
\text { not } \\
\end{array}$ & $\begin{array}{c}\mathbf{3 2 . 3} \\
\text { obser }\end{array}$ & Apr. 22 & 10.2 & 2.3 & 36.0 & & $\mathbf{D}$ & \\
\hline
\end{tabular}

$\mathrm{D}$ and $\mathrm{N}$ denote day- and night-time activity respectively.

and also the parameters of any related theoretical meteor showers were derived in the previous section. It is instructive to find real meteor showers with elements close to the theoretically predicted values. We regard the theoretical and observational showers to be similar if the following conditions are satisfied. The positions of the radiants have to be closer than $\pm 15^{\circ}$ in right ascention and $\pm 10^{\circ}$ in declination. The difference in geocentric velocity has to be less than $5 \mathrm{~km} \mathrm{~s}^{-1}$ and the time of maximum activity within 15 days of each other. Finally, we adopted the value of $D_{\mathrm{S}-\mathrm{H}}$ is the Southworth \& Hawkins criterion given earlier has to be less than 0.25 .

The data from the following published catalogues of observed meteor and fireball showers was used to find observational counterparts to the theoretical showers: Cook (1973) (indicated by C), Kashcheev et al. (1967) (K), Sekanina (1973) (S2), Sekanina (1976) (S3), Gajdosh \& Porubcan (2004) (G), Cannon (2005) (C1), Betlem et al. (2001) (DMS), Porubcan \& Gavajdova (1994) (PG) as well as other data sets circulated privately. The observational counterparts (meteor and fireball showers, and associations) that we found are given in Tables 3-5 (equinox 2000.0). In these tables the numbers and codes of observed showers are given according to the IAU Working List of Meteor Showers.

Matches were found for all the theoretical showers with the exception of one of the theoretical showers associated with NEA 2003MT9 and the values of $D_{\mathrm{S}-\mathrm{H}}$ given in all three tables show values generally significantly less than our adopted critical value, indicating a satisfactory agreement between the predicted and observed showers. The most prominent match is between the active night-time Northern and Southern Iota-Aquariids fireball showers (Gajdosh \& Porubcan 2004) and theoretical showers associated with all three of the NEAs. Matches were also found with the meteor showers Daytime April Piscids and Daytime April Cetids (Kashcheev et al. 1967), while some of the theoretical showers matched a significant number of individual orbits found in the Meteor orbits data center (MODC). By our initial assumption, NEOs associated with meteor showers were to be regarded as being of cometary origin. As all three produce the same matches, and their orbits match each other, it is a reasonable hypothesis that all three are fragments of a larger cometary body that no longer exists.

\section{Conclusions}

Our initial assumption was that NEOs that have meteor showers associated with them should be regarded as being either a dormant cometary nucleus or a surviving fragment from the breakup of a cometary nucleus. We have found three bodies, classified as asteroids, namely 2002JS2, 2002PD11, and 2003MT9, moving on orbits that are very similar to each other and all of which can also be associated with the well documented Northern and Southern Iota-Aquariids fireball showers. Fireballs also indicate that meteoroids larger than those normally associated with meteor showers exist in the stream. All these facts suggest strongly that what we now observe is all that remains of what was once a larger cometary body that no longer exists. It would be extremely instructive to obtain further observations of these three objects in order to ascertain whether or not further signatures of their cometary nature can be located.

Acknowledgements. The authors would like to express their gratitude to the anonymous reviewer for useful comments which improved the paper. We gratefully acknowledge the support of the International Science and Technology Center Project T-1629, without which the work could not have been carried out.

\section{References}

Arter, T. R., \& Williams, I. P. 1997, MNRAS, 289, 721

Asher, D. J., Clube, S. V. M., \& Steel, D. I. 1993, MNRAS, 264, 93

Babadzhanov, P. B. 2001, A\&A, 373, 329

Babadzhanov, P. B., \& Obrubov, Yu. V. 1992, Cel. Mech. Dyn. Astron., 54, 111 Babadzhanov, P. B., \& Williams, I. P. 2007, in Near-Earth Objects our Celestial Neighbors: Opportunity and Risk, ed. A. Milani, G. B. Valsecchi, \& D. Vokrouhlicky (Cambridge: Cambridge University Press), Proc. IAU Symp., 236, 135

Babadzhanov, P. B., Williams, I. P., \& Kokhirova, G. I. 2008a, MNRAS, 386, 1436

Babadzhanov, P. B., Williams, I. P., \& Kokhirova, G. I. 2008b, A\&A, 479, 249

Betlem, H., et al. 2001, Dutch Meteor Society Photographic Orbit Database (Dutch Meteor Society)

Binzel, R. P., \& Lupishko, D. F. 2006, in Asteroids, Comets, Meteors, ed. D. Lazzaro, S. Ferraz-Mello, \& J. A. Fernandez (Cambridge: Cambridge University Press), Proc. IAU Symp., 229, 207

Bowell, E., \& Lumme, K. 1982, in Asteroids, ed. T. Gehrels (Tucson: Univ. Ariz. Press), 132

Cannon, E. 2005

http://web.austin.utexas.edu/edcannon/aka-date-htm 
Cook, A. F. 1973, in Evolutionary and Physical Properties of Meteoroids, ed. C. L. Hemenway, P. M. Millman, \& A. F. Cook, Washington, DC, NASA SP-319, 183

Everhart, E. 1974, Celest. Mech., 10, 35

Fitzsimmons, A., Dahlgren, M., Lagerkvist, C.-I., Magnusson, P., \& Williams, I. P. 1994, A\&A, 282, 634

Gajdosh, S., \& Porubcan, V. 2004, in Dymanics of Populations of Planetary Systems, ed. Z. Knezevic \& A. Milani (Cambridge University Press), Proc. IAU Coll., 197, 393

Goryachev, N. N. 1937, Halphen's Method for Calculation of Planetary Secular Perturbations and its Application to Ceres, Krasnoe Znamya, Tomsk

Jenniskens, P. 2004, AJ, 127, 3018

Jenniskens, P. 2006, Meteor showers and their parent comets (Cambridge University press)

Jenniskens, P. 2008, Icarus, 194, 13

Jenniskens, P., \& Vaubaillon, J. 2007, AJ, 134, 1037

Jenniskens, P., \& Vaubaillon, J. 2008, AJ, 136, 725

Jewitt, D. C. 1992, in Comets in the Post-Halley Era, ed. R. L. Newburn, et al. (Dordrecht: Kluwer), 19

Kashcheev, B. L., Lebedinets, V. N., \& Lagutin, M. F. 1967, Meteoric Phenomena in the Earths atmosphere (Moscow: Nauka), 260

Kresak, L. 1954, Bull. Astr. Inst. Czecho-Sl., 5, 45

Morbidelli, A., \& Gladman, B. 1998, Meteoritics \& Planetary Science, 33, 999

Obrubov, Yu. V. 1999, in Meteoroids 1998, ed. W. J. Baggaley, \& V. Porubcan, Slovak Ac. of Sci., Bratislava, Proc. of the Intern. Conf., 167
Porubcan, V., \& Gavajdova, M. 1994, Planet. Space Sci., 42, 151

Porubcan, V., Kornos, L., \& Williams, I. P. 2004, Earth, Moon and Planets, 95, 697

Porubcan, V., Kornos, L., \& Williams, I. P. 2006, Contrib. of the Astron. Obs. Skalnate Pleso, 36, 103

Sekanina, Z. 1970, Icarus, 13, 475

Sekanina, Z. 1973, Icarus, 18, 253

Sekanina, Z. 1976, Icarus, 27, 265

Southworth, R. B., \& Hawkins, G. S. 1963, Smith. Contrib. Astrophys., 7, 261

Steel, D. I., Asher, D. J., \& Clube, S. V. M. 1991, MNRAS, 251, 632

Vaubaillon, J., Colas, F., \& Jorda, L. 2005, A\&A, 439, 761

Whipple, F. L. 1951, ApJ, 113, 464

Williams, I. P. 1993, in Meteors and their Parent Bodies, ed. J. Stohl, I. P. Williams, Slovak Ac. of Sci., Bratislava, 8

Williams, I. P. 1995, Earth, Moon \& Planet, 68,1

Williams, I. P. 1997, A\&G, 38, 23

Williams, I. P. 2001, Cel. Mech. Dyn. Astron., 81, 103

Williams, I. P. 2004, WGN, The journal of IMO, 32, 11

Williams, I. P., \& Wu, Z. 1993, MNRAS, 262, 231

Williams, I. P., Ryabova, G. O., Baturin, A. D., \& Chernetsov, A. M. 2004, MNRAS, 355, 1171

Wu, Z., \& Williams, I. P. 1992, MNRAS, 259, 617

Wu, Z., \& Williams, I. P. 1993, MNRAS, 264, 980 